\title{
Multi Objective Optimization of Production-Distribution Problem under Fuzzy Random Environment
}

\author{
Muhammad Nazim*, Muhammad Hashim and Jiuping $X u$
}

Uncertainty Decision-Making Laboratory, Sichuan University, Chengdu 610064, P. R. China

\begin{abstract}
In today competitive trade world, the managers most important concern are to make their firms viable and looking effective tools for decision making in the complex business world. This paper describes a hierarchical multi objective production-distribution planing problem under fuzzy random environment. A mathematical model is presented to describe the purpose problem. To deal the uncertain environment, the fuzzy random variables are first transformed into trapezoidal fuzzy numbers, and by using the expected value operation, the trapezoidal fuzzy numbers are subsequently defuzzified. For solving the multi-objective problem a weighted sum base genetic algorithm is applied. Finally, the result of a numerical example is presented to demonstrate the practical and efficiency of the optimized model.
\end{abstract}

Keywords: Multi-objective optimization; Fuzzy lead-time; Fuzzy inventory cost parameters; Inventory Planing; Interactive fuzzy decision making method

\section{Introduction}

A supply chain contains all activities that transform raw materials to final products and deliver them to the customers. Productiondistribution (PD) planning is most important operational function in a supply chain. In today competitive environment, it is required to plan the products, manufactured and distribution, also need for higher efficiency, lower production cost and maximize the customer satisfaction. In general PD problems in supply chains, the decision maker attempts to achieve the following (a) set overall production levels for each product category for each source (manufacturers) to meet fluctuating or uncertain demand for various destinations (distributors) over the intermediate planning horizon, and (b) make right strategies regarding production, subcontracting, back ordering, inventory and distribution levels, and thus determining appropriate resources to be used $[1,2]$. Several methods and algorithms have been developed to solve various PD problems in certain environments [3-5].

In real world $\mathrm{PD}$ problems, however, related environmental coeffcients and parameters, including market demand, available labor levels and machine capacities, and cost/time coeffcients, are often imprecise/fuzzy because of some information being incomplete or unobtainable. It is critical that the satisfying goal values should normally be uncertain as the cost coeffcients and parameters are imprecise/fuzzy in practical PD problems [2,6]. The practical PD problems generally have conflicting goals in term of the use of organizational resources, and these conflicting goals must be simultaneously optimized by the decision makers in the framework of imprecise aspiration levels $[7,8]$. The conventional deterministic techniques cannot solve all integrating PD programming problems in uncertain environments. PD planning is a core issue influencing the producer, distributor and customer. The importance of PD planning has already been recognized $[4,5,9]$ and structure and different views of PD planning have been proposed in a great deal of research [10-16].

The uncertainty in PD system is widely recognized because uncertainties exist in a variety of system components. As a result, the inherent complexity and stochastic uncertainty existing in real world PD decision making have essentially placed them beyond conventional deterministic optimization methods. While, modeling a productiondistribution problem, production costs, purchasing, selling prices, transportation cost, delivery time and demand of products in the objectives and constraints are defined to be confirmed. However, it is seldom so in the real life. For example, holding cost for an item is supposed to be dependent on the amount put in the storage. Similarly, set-up cost also depends upon the total quantity to be produced in a scheduling period, transportation cost depend upon the number of items delivered and scheduling the good network, delivery time also depend upon the production capacity and communication network. So, due to the specific requirements and local conditions, uncertainties may be associated with these variables and the above goals and parameters are normally vague and imprecise, i.e. fuzzy random variable in nature. However, from the previous study review, there appear to be few literature that deal with the uncertainty environment using both fuzziness and randomness in supply chain PD planning problem. Kwakernaak $[17,18]$ introduced a mathematical model by using fuzzy random variables, which was later developed more clearly by Kruse and Meyer [19]. In the Kwakernaak/Kruse and Meyer approaches, fuzzy random variables is viewed as a fuzzy perception/observation/report of a classical real-valued random variable. Xu and Pei [20] proposed a construction supply chain management PD planning; a bi-level model with demand and variable production costs with both fuzzy and random varieties is developed. From a probability space fuzzy random variable is a measurable function to a collection of fuzzy variables, so, roughly speaking, a fuzzy random variable is a random variable that takes fuzzy values. In this paper, for production-distribution planning, a bi-level multi objective model with demand, production costs, selling price and transportation costs all are considered as a fuzzy random.

This paper contributes to current research as follows: first, a multiobjectives model is proposed which considers two objective functions in large-scale industry which solve PD planning problem. In addition, fuzzy random variables are used to describe the demand, variable

*Corresponding author: Muhammad Nazim, Uncertainty Decision-Making Laboratory, Sichuan University, Chengdu 610064, P. R. China, Tel: +8615928414454; E-mail: mailto:nazimscu@gmail.com

Received February 22, 2014; Accepted June 13, 2014; Published June 24, 2014

Citation: Nazim M, Hashim M, Xu J (2014) Multi Objective Optimization of Production-Distribution Problem under Fuzzy Random Environment. Global J Technol Optim 5: 161. doi: 10.4172/2229-8711.1000161

Copyright: @ 2014 Nazim M, et al. This is an open-access article distributed under the terms of the Creative Commons Attribution License, which permits unrestricted use, distribution, and reproduction in any medium, provided the original author and source are credited. 
production costs, transportation cost and delivery time, which assists decision makers to make more effective and precise decisions. In the following sections of this paper is designed as follow. In section 2 multi objective problem description and motivation of using fuzzy random variables are described. A mathematical model is used to optimized the production-distribution planning is explained in section 3. In section 4 fuzzy random simulation based genetic algorithm is explained. A numerical example is parented in section 5 to show the significance of proposed model. At the end conclusions are given in section 6.

\section{Multi Objective Problem Description}

This paper considers multi-objective PD problems under uncertain environment. Assume that the decision maker attempts to determine the integrating $\mathrm{PD}$ plan for $\mathrm{K}$ types of homogeneous commodities from $\mathrm{L}$ sources (factories) to $\mathrm{M}$ destinations (distribution centers) to satisfy the market demand. Every source has a supply of the commodity available to distribute to various destinations, and each destination has its forecast demand for the commodity to be received from the sources. The estimate demand, unit cost coefficients, and delivery time are normally fuzzy random owing to incomplete and unobtainable information over the intermediate planning horizon. This work focuses on developing an expected programming method for optimizing the PD plan in fuzzy random environments.

\section{Motivation for Employing Fuzzy Random Variables in Production-Distribution planning}

The need to describe uncertainty in PD planning is widely acknowledged because uncertainties exist in a variety of system components and a linkage to the regulated policies. In PD the source of the uncertainty mainly has four aspects in the PD planning: production cost; transportation cost, market demand and delivery time. Uncertainty in production mainly exist on the reliability of the production system. Such as; machine fault, change in input prices, executive deviation of the plan etc. Similarly, uncertainty exist in the market demand of the product. Randomness exist in the market demand because of change in product price and season, disaster, market competitors influence etc. Uncertainty also exist in transportation cost of product, transfer to the sale markets. Such as change in flue price, market distance from distribution center, quantity of order etc. Uncertainty may exist in the delivery time because of labor strike, machine working and shortage of components that help in manufacturing the products etc. Generally we define out the uncertainty first with the help of sampling analysis on the base of statistical data when considering the production cost, market demand, transportation cost and delivery time. Then we can value them and make fuzzy random variables with the help of expert experiences. In such a case of study, because it is very difficult to estimate the accurate value of all these fuzzy random variables. It is mostly defined by giving a range in which the most possible value is considered as a random variable, i.e, viz $(a, \rho, b)$ On the basis of statistics characteristics it is found that the most possible value of all these fuzzy random variables follow a normal distribution, i.e, $\rho \sim N(\mu, \sigma 2)$. To deal this situation the triangular fuzzy random variables $(a, \rho, b)$, where $\rho \sim N(\mu, \sigma 2)$ is applied to deal with these uncertain parameters by combining fuzziness and randomness. As a consequence, it is appropriate to consider production cost, product demand, transportation cost and delivery time as a fuzzy random variables.

\section{Modeling}

In this section, a multi objective programming model for the PD planning considering fuzziness and randomness is constructed. The
Notations and mathematical description of the problem is given as follows:

\section{Parameters and Notations}

\section{Index Sets}

$k$ Index for source, for all $k=1,2 \ldots, K$

$l$ Index for kind of product, for all $l=1,2 \ldots ., L$

$j$ Index for destinations of delivery, for all $j=1,2 \ldots ., J$

\section{Parameters}

$\boldsymbol{U}_{w}$ Maximum amount of inventory that can be store in warehouse

$\tilde{\bar{c}}_{k l}$ Fuzzy random total cost of production per unit for product $l$ by source $k$

\section{$Y_{k l j}$ Inventory level of product $k$ by source $l$ for destination $j$}

$h_{k l}$ Inventory holding cost per unit of product $k$ by source $l$

$\tilde{\bar{t}}_{k l j}$ Fuzzy random delivery cost per unit of product $k$ by source $l$ to destination center $j$

$S_{k l}$ Setup cost per unit of product $l$ by source $k$

$\tilde{\bar{P}}_{k l}$ Fuzzy random production cost per unit of product $l$ by source $k$

$r_{k l}$ Rate of production of product $l$ by source $k$

$M_{k}$ Maximum level of production of source $k$

$P_{t}$ Delivery time period lengths

$\tilde{\bar{T}}_{k j}$ Fuzzy random per unit delivery time of product $l$ by source $k$ for destination $j$

$I_{k l}$ is per unit inventory holding coast of product $l$ by source $k$

\section{Decision Variables}

$X_{k l}$ Production volume of product $l$ by source $k$

$p_{t}$ Delivery time periods length

\section{Mathematical Model Formulation}

The multi objective optimization model of PD planning under fuzzy random environment is mathematically formulated as follow:

Objective function 1: The first objective of $\mathrm{PD}$ plan is to minimize the total cost. The total cost of PD planning is composed by three parts namely total production cost which included regular production cost and setup cost, inventory holding cost and product delivery cost. The mathematical expression is as follow.

$$
\begin{aligned}
& \min F_{1}=\sum_{k} \sum_{l} X_{k l} \tilde{\bar{c}}_{k l}+\sum_{k} \sum_{l} I_{k l} h_{k l}+\sum_{k} \sum_{l} \sum_{j} y_{k l j} \tilde{\bar{t}}_{k l j} \\
& \text { Where } \\
& \tilde{\overline{\mathrm{c}}}_{k l}=\sum_{k} \sum_{l} S_{k l}+\sum_{k} \sum_{l} P_{k l} \quad \text { (3.2) }
\end{aligned}
$$

Where $\boldsymbol{X}_{k l}$ is the amount of product $l$ produced by the plant $k$ and $\tilde{\bar{c}}_{k l}$ is per unit cost of product $l$ of plant $k . I_{k l}$ is per unit inventory holding coast and $h_{k l}$ is the total number of items of product hold in warehouse. Where as $y_{k l j}$ is the amount of product transfer to the $j$ destination and $\overline{\bar{t}}_{k l j}$ is per unit transportation cost.

Constraints: The transformation of product should satisfy the minimum demand of $j$ destination to assure the customer satisfaction. So that, sum of total available product to transfer is greater than sum of 
total demand of $j$ destination.

$$
\sum_{k} \sum_{l} \sum_{j} y_{k l j} \geq\left[E\left(\tilde{\bar{D}}_{k l j}\right)\right]
$$

Where $y_{k l j}$ is the available amount of product $l$ by the source $k$ for distinction $j$ and $\left[E\left(\bar{D}_{k l j}\right)\right]$ is the expected value demand of product by destination $j$.

The sources (plants) are working at maximum level

$$
\sum_{k} \sum_{l} r_{k l} X_{k l} \leq M_{k}
$$

Inventory level of product is less than the upper bound of warehouses

$$
0 \leq \sum_{k} \sum_{l} I_{k l} \leq U_{w}
$$

Objective function 2: The second objective of PD plan is to minimize the delivery time, which is mathematically formulated as follow.

$$
\operatorname{minF}_{2}=\sum_{k} \sum_{l} \sum_{j} y_{k l j} \tilde{\bar{T}}_{k l j}
$$

Where $y_{k l}$ the amount of product is $l$ transfer by plant $k$ to destination $j$ and $\bar{T}_{k l j}$ is the expected transfer time per unit to destination $j$.

Constraints: The total delivery time of product must be less than period time

$$
\sum_{k} \sum_{l} \sum_{j} y_{k l j} \tilde{\bar{T}}_{k l j}
$$

From the above discussion, by the integration of Eq. 3.1-3.7 a fuzzy random multi objective expected value model for production-distribution can be formulated as follow,

$$
\left\{\begin{array}{l}
\min F_{1}=\sum_{k} \sum_{l} X_{k l}\left[E\left(\tilde{\bar{c}}_{k l}\right)\right]+\sum_{k} \sum_{l} I_{k l} h_{k l}+\sum_{k} \sum_{l} \sum_{j} y_{k l j}\left[E\left(\tilde{\tilde{t}_{k j}}\right)\right] \\
\min F_{2}=\sum_{k} \sum_{l} \sum_{j} y_{k l}\left[E\left(\tilde{\bar{T}}_{k l j}\right)\right] \\
\sum_{k} \sum_{l} \sum_{j} y_{k l j} \geq\left[E\left(\tilde{\bar{D}}_{k l j}\right)\right] \\
\sum_{k} \sum_{l} r_{k l} X_{k l} \leq M_{k} \\
0 \leq \sum_{k} \sum_{l} I_{k l} \leq U_{w} \\
\sum_{k} \sum_{l} \sum_{j} y_{k l j}[E(\tilde{\overline{\bar{T}}})] \leq p_{t}
\end{array}\right.
$$

\section{Solution Method}

To solve the previous multi objective PD planning problem, four step are proposed. First, a fuzzy random variables transform into fuzzy trapezoidal numbers. Secondly, fuzzy simulation is applied to calculate the expected value of objective functions. Third, weighted sum method is used to transformed the multi objective into single objective. At the end a genetic algorithm is proposed to solve the above describe multi objective problem (Figure 1).

\section{Dealing with Fuzzy Random Variables}

Generally we know that, it is difficult to directly obtain an optimal solution of fuzzy random variables. Therefore, the fuzzy random variables convert into deterministic ones by the proposed transformation method. At First, the fuzzy random variables are transformed into fuzzy

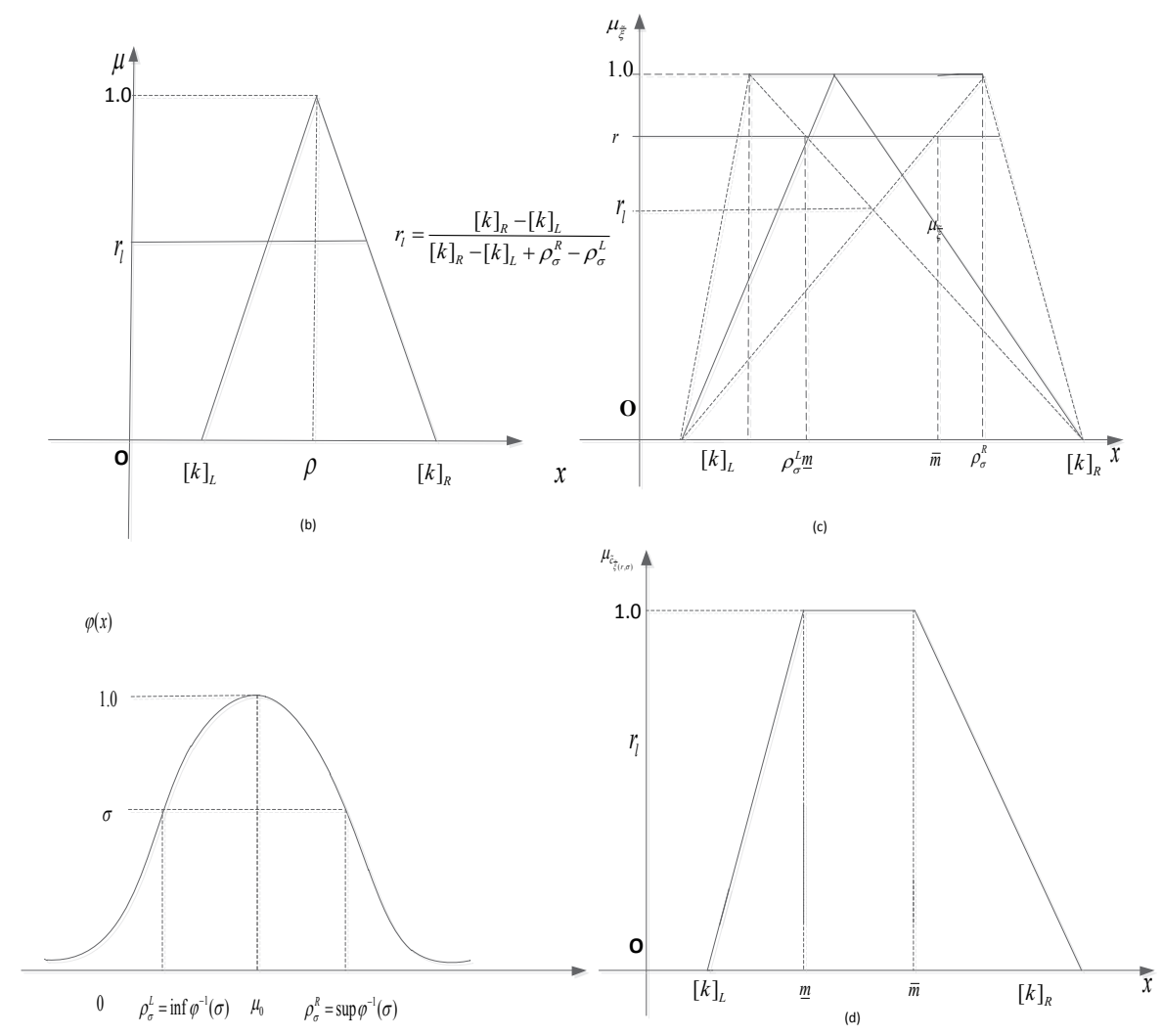

Figure 1: Transforming fuzzy random variable to trapezoidal fuzzy number. 
numbers, and then Heilpern [21] expected value operator is applied to drive the deterministic variables.

\section{Transformation of fuzzy numbers variables into fuzzy numbers}

Total Production Cost: Generally, fuzzy random parameters are denoted as, where is a random variable with a probability density function of. It is supposed approximately follow a normal distribution, then

$$
\varphi_{\rho}(x)=\frac{1}{\sqrt{2 \pi \sigma_{0}}} e-\left(x-\mu_{0}\right)^{2} / 2 \sigma_{0}^{2}
$$

Suppose that $\sigma$ is a given probability level of random variable and $\sigma \in\left[0, \sup \varphi_{\rho}(x)\right], r$ is a given possibility level for the fuzzy variable and $r \in\left[r_{p} 1\right]$ where

$$
r_{l}=\left[\frac{[m]_{R}-[m]_{L}}{[m]_{R}-[m]_{L}+\rho_{\sigma}^{R}-\rho_{\sigma}^{L}}\right]
$$

both of them reflecting the decision maker's degree of optimism. For an easy description, the probability level and the possibility level are called $\sigma$ and $r$ respectively. The transformation method consists of the following steps:

(1) Through historical data and professional experience using statistical laws, estimate the parameters $[\mathrm{m}]_{L},[\mathrm{~m}]_{R}, \mu_{0}$, and $\sigma_{0}$

(2) Obtain the decision maker's degree of optimism, i.e., the values of probability level $\sigma \in\left[0\right.$, sup $\left.\varphi_{\rho}(x)\right], r$ and possibility level $r \in\left[r_{p} 1\right]$, where

$$
r_{l}=\left[\frac{[m]_{R}-[m]_{L}}{[m]_{R}-[m]_{L}+\rho_{\sigma}^{R}-\rho_{\sigma}^{L}}\right]
$$

Which are often determined by using a group- decision making approach.

(3) Let $\rho_{\sigma}$ be the $\sigma$ cut of the random variable $\rho(c)$, that is $\rho_{\sigma}=\left[\rho_{\sigma}^{L}, \rho_{\sigma}^{R}=\left\{x \in R \mid \varphi_{\rho(x)} \geq \sigma\right]\right.$ then the value of $\rho_{\sigma}^{L}$ and $\rho_{\sigma}^{R}$ can be expressed as

$$
\begin{aligned}
& \rho_{\sigma}^{L}=\inf \left\{x \in R \mid \varphi_{\rho(x)} \geq \sigma\right\}=\inf \varphi_{\rho}^{-1}(\sigma)=\mu_{0}-\sqrt{-2 \sigma_{0}^{2} \ln \sqrt{2 \pi} \sigma_{0} \sigma} \\
& \rho_{\sigma}^{R}=\inf \left\{x \in R \mid \varphi_{\rho(x)} \geq \sigma\right\}=\inf \varphi_{\rho}^{-1}(\sigma)=\mu_{0}+\sqrt{-2 \sigma_{0}^{2} \ln \sqrt{2 \pi} \sigma_{0} \sigma}
\end{aligned}
$$

(4) Transform the fuzzy random variable $\tilde{\bar{x}}=\left([k]_{L}, \rho(c),[k]_{R}\right)$ into the $(r, \sigma)$ level trapezoidal fuzzy number $\tilde{c}_{\tilde{\xi}(r, \sigma)}$ by using the following equation:

$$
\tilde{\bar{\xi}}=\tilde{c}_{\tilde{\xi}(r, \sigma)}=\left([m]_{L}, \underline{m}, \bar{m},[m]_{R}\right),
$$

Where

$$
\begin{aligned}
& \underline{m}=[m]_{R}-r\left([m]_{R}-\rho_{\sigma}^{L}\right)=[m]_{R}-r\left[[m]_{R}-\mu_{0}+\sqrt{-2 \sigma_{0}^{2} \ln \sqrt{2 \pi} \sigma_{0} \sigma}\right] \\
& \bar{m}=[m]_{L}+r\left(\rho_{\sigma}^{R}-[m]_{L}\right)=[m]_{L}+r\left[\mu_{0}-[m]_{L 0}+\sqrt{-2 \sigma_{0}^{2} \ln \sqrt{2 \pi} \sigma_{0} \sigma}\right]
\end{aligned}
$$

$\tilde{\bar{\xi}}$ can be specified by $\tilde{c}_{\tilde{\xi}(r, \sigma)}=\left([m]_{L}, \underline{m}, \bar{m},[m]_{R}\right)$ with the following membership function:

$$
\mu_{\tilde{c}_{\tilde{\xi}(r, \sigma)}}=\left\{\begin{array}{l}
0 \text { for } x \prec[m]_{L}, x \succ[m]_{R} \\
\frac{x-[m]_{L}}{m-[m]_{L}} \text { for }[\mathrm{m}]_{L} \leq x \prec \underline{m} \\
1 \text { for } \underline{\mathrm{m}} \leq x \leq \bar{m} \\
\frac{[m]_{R}-x}{[m]_{R}-\bar{m}} \text { for } \overline{\mathrm{m}} \leq x \prec[m]_{R}
\end{array}\right.
$$

The transformation process of fuzzy random variable ${ }^{-}$to the $(r, \sigma)$ level trapezoidal fuzzy number $\tilde{\mathcal{c}}_{\tilde{\xi}(r, \sigma)}$ is described in Eq.(3.8-3.16)

Let $\tilde{\bar{t}}_{i k}$ transportation cost $\tilde{\bar{D}}_{i k}$ demand of product and $\tilde{\bar{T}}_{i k}$ per unit delivery time of product are also fuzzy random variables. Based on the previous method described for $\tilde{\bar{c}}_{i k}$ total cost of production, can be transformed into $(r, \sigma)$ level trapezoidal fuzzy numbers as follow;

$$
\tilde{\bar{c}}_{k l}(m)=\tilde{c}_{k l}(m)_{(r, \sigma)}=\left(\left[c_{k l}(m)_{L}\right], c_{k l}(\underline{m}), c_{k l}(\bar{m}),\left[c_{k l}(m)_{R}\right]\right)
$$$$
\tilde{\bar{T}}_{k j}(m)=\tilde{T}_{k l j}(m)_{(r, \sigma)}=\left(\left[T_{k l j}(m)_{L}\right], T_{k l j}(\underline{m}), T_{k l j}(\bar{m}),\left[T_{k l j}(m)_{R}\right]\right)
$$

$\tilde{\bar{t}}_{k l}(m)=\tilde{t}_{k l}(m)_{(r, \sigma)}=\left(\left[t_{k l}(m)_{L}\right], t_{k l}(\underline{m}), t_{k l}(\bar{m}),\left[t_{k l}(m)_{R}\right]\right)$

$\tilde{\bar{D}}_{k l j}(m)=\tilde{D}_{k l j}(m)_{(r, \sigma)}=\left(\left[D_{k l j}(m)_{L}\right], D_{k l j}(\underline{m}), D_{k l j}(\bar{m}),\left[D_{k l j}(m)_{R}\right]\right)$

\section{Expected value model}

For computing the expected value of the above described $(r, \sigma)$ - level trapezoidal fuzzy variables, a new fuzzy measure with an optimistic pessimistic adjusting index is introduced to characterize real- life problems. The definition of this fuzzy measure Me which is a convex combination of Pos and Nec, can be found in Xu and pei [20], whereas the basic knowledge for measures Pos and Nec can be seen in Dubois and Prade (1998)

Let $\tilde{x}=\left(r_{1}, r_{2}, r_{3}, r_{4}\right)$ denote a trapezoidal fuzzy variable. In fact, in real-world problems, especially the inventory problem in large-scale construction projects, the case when $\left[r_{1} \succ 0\right]$ is often encountered. Based on the definition and properties of the expected value operator of a fuzzy variable using the measure $M e$ [20], if the fuzzy random variable $\tilde{m}$ is transformed into the $(r, \sigma)$ - level trapezoidal fuzzy variable $\tilde{x}_{r, \sigma}=[m]_{L}, \underline{m}, \bar{m},[m]_{R}$ where $[m]_{\mathrm{L}}$ then the expected value of $\tilde{m}_{(r, \sigma)}$ should be

$$
E^{M e}\left[\tilde{m}_{r, \sigma}\right]=\frac{1-\lambda}{2}\left([m]_{L}+\underline{m}\right)+\frac{\lambda}{2}\left(\bar{m}+[m]_{R}\right)
$$

Based on the above methods, the expected value of the $(r, \sigma)$ level trapezoidal fuzzy variable involved in each objective function and state equation can be calculated by Equation (21).

\section{Fuzzy Random Simulation}

The fuzzy simulation is used to deal with those which cannot be converted into crisp ones. Xu and pei [20] put forward a fuzzy random simulation combining stochastic simulation and fuzzy simulation to solve these problems. In this section, we have proposed such kind of simulation is used to determine the equivalent value of the objective functions dealt with by the expected operator.

Step 1: Set E=0;

Step 2: Generate independently random numbers sample $\omega$ from $\Omega$ according to the probability measure $P$;

Step 3: $E \leftarrow e+E[\tilde{\bar{F}}(\omega)]$, where $E[\tilde{\bar{F}}(\omega)]$ is calculated by the fuzzy simulation as following sub-steps;

Step 3.1: Set $\mathrm{E}=0$;

Step 3.2: Randomly generate $u_{j}=\left(u_{1 j}, u_{2 j}, \ldots, u_{\mathrm{nj}}\right)$ from the $\varepsilon$ - level sets of $\omega_{1}, \omega_{2}, \ldots, \omega_{\mathrm{n}}$ and represent $u_{\mathrm{j}}=\left(u_{1 j}, u_{2 j}, \ldots, u_{\mathrm{nj}}\right), j=1,2, \ldots, m$ respectively, where $\varepsilon$ is a sufficiently small number.

Step 3.3: Set $a=f\left(u_{1}\right) \wedge f\left(u_{2}\right) \wedge \ldots \ldots \wedge f\left(u_{m}\right), b=f\left(u_{1}\right) \vee f\left(u_{2}\right) \vee \ldots . \vee f\left(u_{m}\right)$.

Step 3.4: Randomly generate $r$ from $[a, b]$. 
Step 3.5: If $E_{d}[\tilde{\bar{F}}]=e / N$, then $E \leftarrow E+C r\{\hat{\bar{F}}(\omega) \geq r\}$.

Step 3.6: If $r \prec 0$, then $E \leftarrow E-C r\{\hat{\bar{F}}(\omega) \leq r\}$.

Step 3.7: Repeat the 3.4 to 3.6 steps for $N$ times.

Step 3.8: $E[\tilde{\bar{F}}(\omega)]=a \vee 0+b \wedge 0+E .(b-a) / N$.

Step 4: Repeat the second to fourth steps $N$ times;

Step 5: $E_{d}[\tilde{\bar{F}}]=e / N$

\section{Weighted Sum Method}

The weight sum method is one of the techniques which is mostly applied to solve the multi-objective programming problem. By applying the weighted sum method we can convert the multi objective into single objective giving the weight of each objective function.

Assume that the related weight of the objective function $f_{i}(x)$ is $w_{i}$ such that $\sum_{i=1}^{m} w_{i} f_{i}=1$ and $w_{i} \geq 0$. So we can construct the evaluation

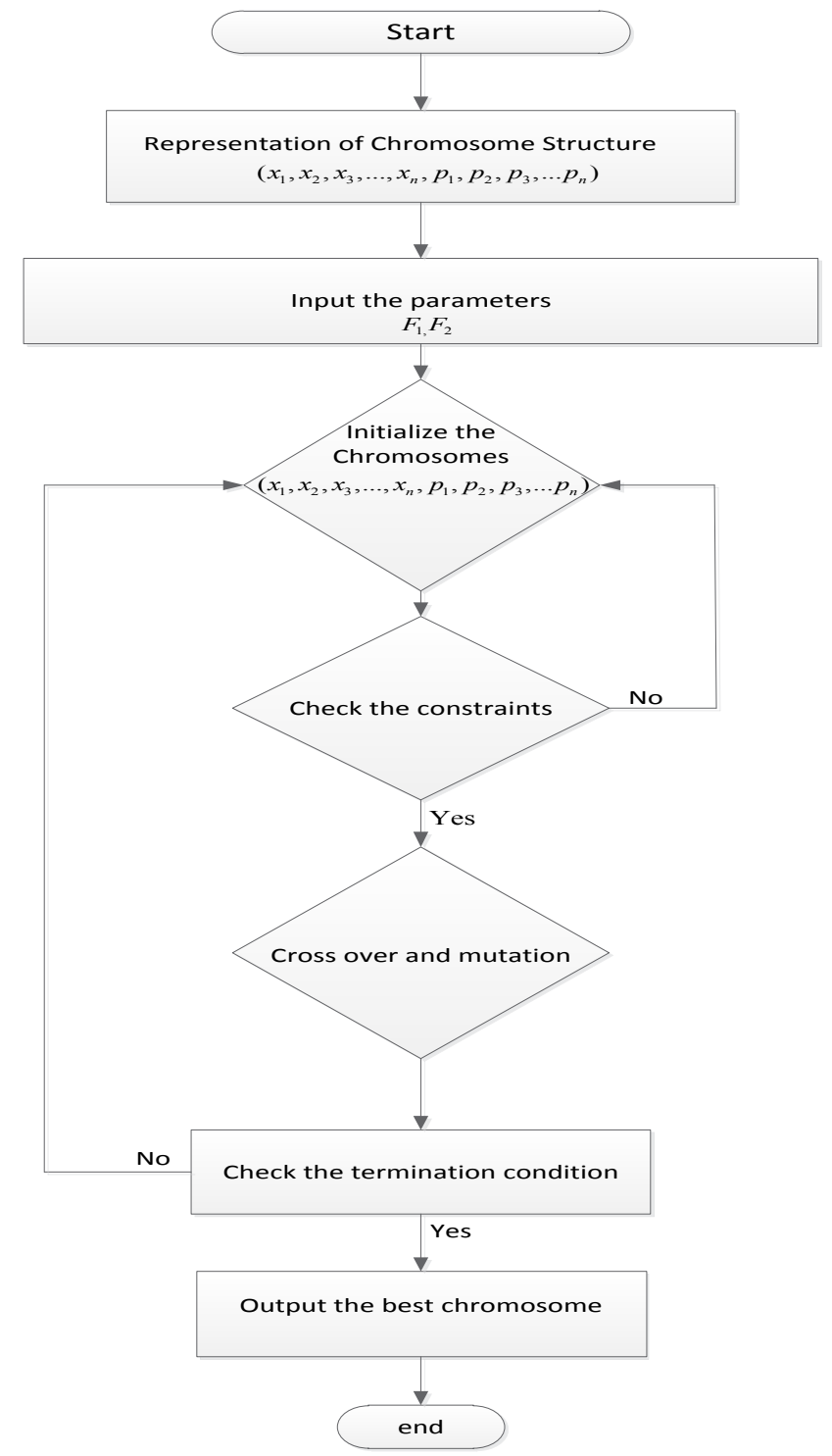

Figure 2: GA process diagram. function as follows,

$$
u(f(x))=\sum_{i=1}^{m} w_{i} f_{i}(x)=w^{t} f(x)
$$

Where $w_{i}$ express the importance of the objective functions $f_{i}(x)$ for decision maker. Then we get the following weight problem

$$
\min _{x \in X} u(f(x))=\min _{x \in X} \sum_{i=1}^{m} w_{i} f_{i}(x)=\min _{x \in X} w^{t} f(x)
$$

\section{Genetic Algorithm}

In this subsection we have applied a stochastic search methods for optimization problems based on the mechanics of natural selection and natural genetics, genetic algorithms (GAs), which have received remarkable attention regarding their potential as a novel approach to multi objective optimization problems. GAs does not need many mathematical requirements and can handle any types of objective functions and constraints. GAs (Figure 2) have been well discussed and summarized by several authors, e.g., Holland [22], Goldberg [23], Michalewicz [24], Fogel [25], Gen and Cheng [16], Liu [26].

This section attempts to present a fuzzy random simulation and weighted sum method-based genetic algorithm to obtain a solution of multi objective programming with fuzzy random coefficients

(1) Representation of chromosome structure: We use a vector $\mathrm{x}=$ $F_{1} F_{2}$ as a chromosome to represent a solution to the optimization of the proposed problem.

(2) Handling the constraints: To ensure the chromosomes generated by genetic operators are feasible, we can use the technique of fuzzy random simulation to check them.

(3) Initializing process of Chromosomes: Suppose that the DM is able to predetermine a region which contains the feasible set. Generate a random vector $v$ from this region until a feasible one is accepted as a chromosome. Repeat the above process $N_{\text {pop-size }}$ times, and then we have $N_{\text {pop-size }}$ initial feasible chromosomes $x^{1}, x^{2}, x^{3}, \ldots, N_{p o p-s i z e}, p^{1}, p^{2}, p^{3}, \ldots, N_{\text {pop-size }}$

(4) Evaluation function: The regret value of each chromosome $v$ is calculated, and then the fitness function of each chromosome is computed by

$$
\operatorname{eval}(v)=\sum_{k=1}^{m} \frac{E\left[f_{k}(v, \xi)\right]-z_{k}^{\max }}{z_{k}^{\max }-z_{k}^{\min }}
$$

(5) Selection process: The selection process is based on spinning the roulette wheel $N_{\text {pap-size }}$ times. Each time a single chromosome for a new population is selected in the following way: Calculate the cumulative probability $q_{i}$ for each $v^{i}$

$$
q_{0}=0, \quad \mathrm{q}_{i}=\sum_{j=1}^{i} p_{j}, i=1,2, \ldots N_{\text {pop-size }} \text { where } p_{j}=\frac{\operatorname{eval}\left(v_{j}\right)}{\sum_{k}^{N_{\text {pop }- \text { ste }}} \operatorname{eval}\left(v_{k}\right)} \text {. }
$$

Generate a random number $r \in[0,1]$ if $q_{i=1}$ p $r \leq q_{i}, i=1,2, \ldots N_{p o p-s i z e}$ then selection chromosome $v_{i}$ Repeat this process $N_{\text {pop-size }}$ times, then $N_{\text {pop-size }}$ copies of chromosomes will be obtained.

(6) Crossover operation: Generate a random number $c$ from the open interval $(0,1)$ and the chromosome $v_{i}$ is selected as a parent provided that $\left[c \mathrm{p} P_{c}\right]$ where parameter $P_{c}$ is the probability of crossover operation. Repeat this process $N_{\text {pop-size }}$ times and $P_{c} \cdot N_{\text {pop-size }}$ chromosomes are expected to be selected to undergo the crossover operation. The crossover operator on $v^{1}$ and $v^{2}$ will produce two children $y^{1}$ and $y^{2}$ as follow

$$
y^{1}=c v^{1}+(1-c) v^{2}, y^{2}=c v^{2}+(1-c) v^{1}
$$


(7) Mutation operation: Similar to the crossover process, the chromosome $v^{i}$ is selected as a parent to undergo the mutation operation provided that random number $\mathrm{m}<P_{m}$, where parameter $P_{m}$ as the probability of mutation operation. $P_{m} \cdot N_{\text {pop-size }}$ chromosomes are expected to be selected after repeating the process $N_{\text {pop-size }}$ times. Suppose that $v^{1}$ is chosen as a parent. Choose a mutation direction $d$ $\epsilon R^{n}$ randomly. Replace $v$ with $v+M . d$ if $v+M . d$ is feasible, otherwise we set $M$ as a random number between 0 and $M$ until it is feasible or a given number of cycles is finished. Here, $M$ is a sufficiently large positive number.

We illustrate the fuzzy random simulation-based genetic algorithm procedure as follows:

The Procedure of combined fuzzy random simulation genetic algorithm

\section{Step 0: Input the parameters, and}

Step 1: Initialize chromosomes whose feasibility may be checked by fuzzy random simulation.

Step 2: Update the chromosomes by crossover and mutation operations and fuzzy random simulation is used to check the feasibility of offspring.

\begin{tabular}{|c|c|c|c|}
\hline \multicolumn{4}{|c|}{$\tilde{\bar{c}}_{1 l}$} \\
\hline \multicolumn{4}{|c|}{$(140, \rho(c), 160)$ where $\rho(c) \sim N(150,4)$} \\
\hline \multicolumn{4}{|c|}{ Table 1: Fuzzy random cost of production. } \\
\hline & $\tilde{\bar{t}}_{k l 2}$ & $\tilde{\bar{t}}_{k l 3}$ & $\tilde{\bar{t}}_{k l 4}$ \\
\hline $\begin{array}{c}(4, \rho(t), 8): \\
\rho(t) \sim \mathrm{N}(7,1)\end{array}$ & $\begin{array}{c}(3.5, \rho(t), 7): \\
\rho(t) \sim \mathrm{N}(6, .8)\end{array}$ & $\begin{array}{c}(4.2, \rho(t), 8.4) \\
\rho(t) \sim \mathrm{N}(7, .9)\end{array}$ & $\begin{array}{l}(4, \rho(t), 6.1) \\
\rho(t) \sim \mathrm{N}(7,1)\end{array}$ \\
\hline
\end{tabular}

Table 2: Fuzzy random cost of transportation $\tilde{\bar{t}}_{k j}$

\begin{tabular}{|c|r|r|r|}
\hline$\tilde{\bar{T}}_{k l 1}$ & $\tilde{\bar{T}}_{k l 2}$ & $\tilde{\bar{T}}_{k l 3}$ & $\tilde{\bar{T}}_{k l 4}$ \\
\hline$(8, \rho(T), 12) ;$ & $(6.5, \rho(T), 10) ;$ & $(8.5, \rho(T), 13) ;$ & $(6, \rho(T), 12) ;$ \\
$\rho(T) \sim \mathrm{N}(11,1)$ & $\rho(T) \sim \mathrm{N}(8.8)$ & $\rho(T) \sim \mathrm{N}(11, .95)$ & $\rho(T) \sim \mathrm{N}(9.5,1)$ \\
\hline
\end{tabular}

Table 3: Fuzzy random delivery time $\tilde{\bar{T}}_{k j}$.

\begin{tabular}{|c|c|c|c|}
\hline$-_{k l}$ & $\tilde{\bar{D}}_{k l 2}$ & $\tilde{\bar{D}}_{k l 3}$ & $\tilde{\bar{D}}_{k l 4}$ \\
\hline $\begin{array}{l}(80, \rho(D), 100) \\
\rho(D) \sim \mathrm{N}(90,4)\end{array}$ & $\begin{array}{c}(60, \rho(D), 90) \\
\rho(D) \sim \mathrm{N}(80,3.5)\end{array}$ & $\begin{array}{c}(85, \rho(D), 100) \\
\rho(D) \sim \mathrm{N}(94,4.1)\end{array}$ & $\begin{array}{c}(65, \rho(D), 90) \\
\rho(D) \sim \mathrm{N}(80,3.5)\end{array}$ \\
\hline
\end{tabular}

Table 4: Fuzzy random demand $\tilde{\bar{D}}_{k l j}$

\begin{tabular}{|c|c|c|c|c|c|c|c|c|}
\hline Notations & $\mathrm{w}_{1}$ & $\mathrm{w}_{2}$ & $\mathrm{w}_{3}$ & $\mathrm{w}_{4}$ & $\mathrm{w}_{5}$ & $\mathrm{w}_{6}$ & $\mathrm{w}_{7}$ & $\mathrm{w}_{8}$ \\
\hline Vlaues & $1 / 8$ & $1 / 8$ & $1 / 8$ & $1 / 8$ & $1 / 8$ & $1 / 8$ & $1 / 8$ & $1 / 8$ \\
\hline Notations & $\mathrm{z}_{11}{ }^{*}$ & $\mathrm{z}_{12}{ }^{*}$ & $\mathrm{z}_{13}{ }^{*}$ & $\mathrm{z}_{14}{ }^{*}$ & $\mathrm{z}_{21}{ }^{*}$ & $\mathrm{z}_{22}{ }^{*}$ & $\mathrm{z}_{23}{ }^{*}$ & $\mathrm{z}_{24}{ }^{*}$ \\
\hline Values & 13420 & 12293 & 11975 & 12864 & 1292 & 1185 & 985 & 1315 \\
\hline
\end{tabular}

Table 5: Optimal objectives values calculated by GA.

\begin{tabular}{|c|c|c|c|c|}
\hline Notations & $\times 11$ & $\times 12$ & $\times 13$ & $\times 14$ \\
\hline Vlaues & 96 & 92 & 98 & 100 \\
\hline Notations & ${ }^{\top} 21$ & ${ }^{\top} 22$ & ${ }^{\top} 23$ & ${ }^{\top} 24$ \\
\hline Values & 8.92 & 6.6 & 8.62 & 5.94 \\
\hline
\end{tabular}

Table 6: Optimal production-distribution plan.
Step 3: Compute the fitness of each chromosome based on the regret value.

Step 4: Select the chromosomes by spinning the roulette wheel.

Step 5: Repeat the second to fourth steps for a given number of cycles.

Step 6: Report the best chromosome as the optimal solution.

\section{Numerical example}

The background of problem, mathematical model of describe problem and solution method have been introduce in section 2, section 3 and section 4 respectively. According to the experts' advice a numerical example is proposed in this section, which illustrates the practical application of the proposed optimized model. Please refer (Tables 1-4)

- Number of production plant (source): 1

- Numbers of distribution places: 4

- Inventory holding cost 2

In the view of final optimal objective functions solution of GA in Table 5, the producer can rationally allocate the production-distribution to save cost and delivery time. We have considered the fuzziness and randomness at the same time when making planning which assist decision makers to make more accurate and well informed planning.

In Table 6, an optimal production-distribution plan is presented which rationally allocate the number of production unit for each distribution center and delivery time of product to market. The proposed solution method is suitable because it's give an efficient solution.

The proposed deterministic model is not suitable method of obtaining an effective solution, because of conflicting nature of the multiple objectives and the vagueness in the information relating to the decision parameters in real-world PD problems. The results describe in Tables 5 and 6 it shows that the interaction of trade-offs and conflicts among dependent objectives. Accordingly, the proposed method satisfies the real application requirement for solving multiobjective PD problems in fuzzy random environments, since it aims to simultaneously minimize total production and distribution costs and total delivery time of product. Additionally, triangular fuzzy random numbers are used for the sake of computational efficiency and ease of data acquisition. In this proposed model we adopt the simplified pattern of triangular distribution with the most possible, most pessimistic and most optimistic values to represent the imprecise total production cost, market demand and delivery time. The pattern of triangular distribution is commonly adopted due to ease in defining the maximum and minimum limit of deviation of the fuzzy random number from its central value. The Figure 3 shows the 100 number of generations, each generation shows the different optimal value of fitness function.

\section{Conclusion}

In this paper, we have proposed the multi objective productiondistribution programming problem with fuzzy random coefficients. For a special type of fuzzy random variables, we have applied a method to transfer into fuzzy number and expected value operator was applied to get the deterministic variables. A fuzzy random simulationbased genetic algorithm using weighted sum method approach which is effective to solve the general fuzzy random multi objective 


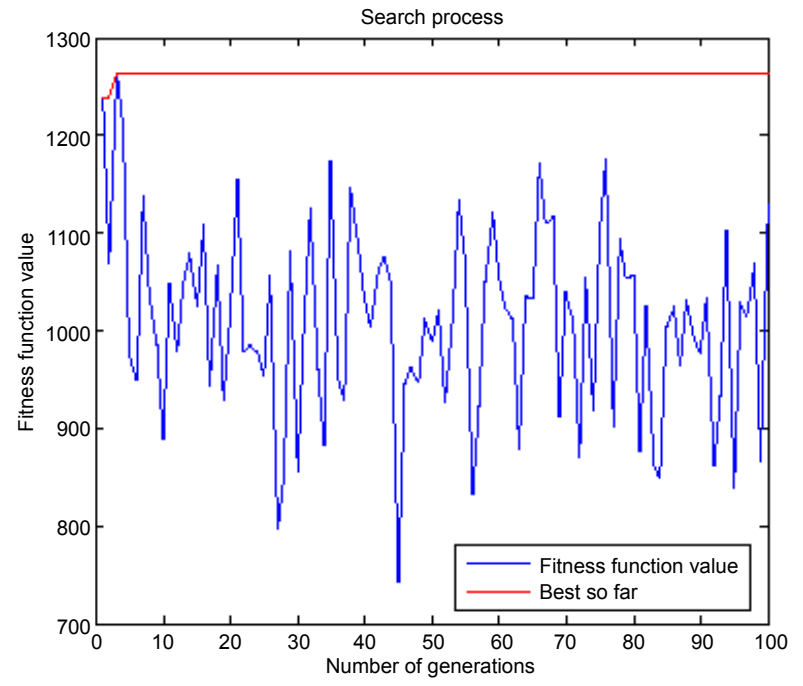

Figure 3: Genetic algorithm search process of multi objective functions fitness.

programming problem. Though the fuzzy random simulation-based genetic algorithm proposed in this paper usually spends more CPU time than traditional algorithms, it is a viable and efficient way to deal with complex optimization problems involving randomness and fuzziness. In the future, fuzzy random simulation-based multi objective genetic algorithm is another field that we will consider.

\section{References}

1. Aliev RA, Fazlollahi B, Guirimov BG, Aliev RR (2007) Fuzzy-genetic approach to aggregate production-distribution planning in supply chain management. Information Sciences 177: 4241-4255

2. Wang RC, Liang TF (2004) Application of fuzzy multi-objective linear programming to aggregate production planning. Comp Indus Enge 46: 1741.

3. Bilgen B, Ozkarahan I (2004) Strategic tactical and operational productiondistribution models: A review. Int J Tech Manage 28: 151-171.

4. Erenguc SS, Simpson NC, Vakharia AJ (1999) Integrated production distribution planning in supply chains: An invited review, Europe $\mathrm{J}$ of Ope Res 115: 219-236.

5. Vidal CJ, Goetschalckx M (1997) Strategic production-distribution models: A critical review with emphasis on global supply chain model. Europe $\mathrm{J}$ of Ope Res 98: 118.

6. Petrovic D, Roy R, Petrovic R (1998) Modelling and simulation of a supply chain in an uncertain environment. Europe J of Ope Res 109: 299-309.
7. Liang TF (2008) Integrating production-transportation planning decision with fuzzy multiple goals in supply chains. Int J of Prod Res 46: 1477-1494.

8. Liang TF, Cheng HW, Chen PY, Shen KH (2011) Application of fuzzy sets to aggregate production planning with multi-products and multi-time periods. IEEE Trans Fuzz Sys 19: 465-477.

9. Hua G, Wang S, Chan CK (2009) A Fractional programming model for internation facility location. J Industrial Manage Opt 5: 629-649.

10. Selim H, Araz C, Ozkarahan I (2008) Collaborative production-distribution planning in supply chain: A fuzzy goal programming approach. Transportation Research Part E: Logistics and Transportation Review 44: 396-419.

11. Liang TF (2008) Fuzzy multi-objective production/distribution planning decisions with multi-product and multi- time period in a supply chain. Comp Indus Eng 55: 676-694.

12. Lee YH, Kim SH (2002) Production-distribution planning in supply chain considering capacity constraints. Comp Industrial Eng 43: 169-190.

13. Averbakh I (2010) On-line integrated production-distribution scheduling problems with capacitated deliveries. European Journal of Operational Research 200: 377-384.

14. Bilgen B, Ozkarahan I (2004) Strategic, tactical and operational productiondistribution models: A review. Int J Tech Manage 28: 151-171.

15. Sarmiento AM, Nagi R (1999) A review of integrated analysis of productiondistribution systems. IEEE Transact 31: 1061-1074.

16. Gen M, Cheng R (1997) Genetic Algorithms and Engineering Design, Wiley, New York

17. Kwakernaak H (1978) Fuzzy random variables-I. Definitions and theorems, Info Sci 15: 1-29.

18. Kwakernaak H (1979) Fuzzy random variables-II, Algorithms and examples for the discrete case. Info Sci 17: 253-278.

19. Kruse R, Meyer KD (1987) Statistics with Vague Data, Reidel Publishing Company, Dordrecht, Germany.

20. Xu J, Pei W (2013) Production-Distribution planning of construction supply chain management under fuzzy random environment for large-scale construction projects, J Indus Manage Opt 9: 31-56.

21. Heilpern S (1992) The expected value of a fuzzy number, Fuzzy Set Syst 47 $81-86$

22. Holland H (1975) Adaption in Natural and Artifical Systems, University of Michigan, Ann Arbor.

23. Goldberg DE (1989) Genetic Algorithms in Search, Optimization and Machine Learning, Addison-Wesley, New York.

24. Michalewicz Z (1994) Genetic Algorithms + Data Structures = Evolution Programs, Springer, New York.

25. Fogel DB (1995) Evolution Computation: Toward a New Philosophy of Machine Intelligence, IEEE Press, Piscataway.

26. Liu B (2002) Theory and Practice of Uncertain Programming, Physica Verlag, New York. 